\title{
Effect of Inducible Nitric Oxide Synthase on Cerebral Blood Flow after Experimental Traumatic Brain Injury in Mice
}

\author{
LESLEY M. FOLEY, ${ }^{1}$ T. KEVIN HITCHENS, ${ }^{1,2}$ JOHN A. MELICK, ${ }^{3}$ HÜLYA BAYIR, $, 3,4$ \\ CHIEN HO, ${ }^{1,2}$ and PATRICK M. KOCHANEK ${ }^{3,4}$
}

\begin{abstract}
Inducible nitric oxide synthase (iNOS) has been suggested to play a complex role in the response to central nervous system insults such as traumatic brain injury (TBI) and cerebral ischemia. In the current study, we quantified maps of regional cerebral blood flow (CBF) using an arterial spin-labeling magnetic resonance imaging (MRI) technique, at 24 and $72 \mathrm{~h}$ after experimental TBI in iNOS knockout (KO) and wild-type (WT) mice. Our hypothesis was that iNOS would contribute to the level of $\mathrm{CBF}$ at $72 \mathrm{~h}$ after experimental TBI in mice. Comparing anatomical brain regions of interest (ROIs) at 24-h post controlled cortical impact (CCI), there were significant reductions in CBF in the hemisphere, cortex, and contusion-rich area of the cortex of injured animals versus naive, regardless of genotype. Regional assessment of $\mathrm{CBF}$ at $72 \mathrm{~h}$ after injury demonstrated that recovery of CBF was reduced in the ipsilateral hippocampus, thalamus, and amygdala/piriform cortex in iNOS KO versus WT mice by $26 \%, 15 \%$, and $21 \%$, respectively; this attenuated recovery was restricted to structures outside the contusion. These regions with reduced CBF in iNOS KO mice represented ROIs where CBF in the WT was either numerically or statistically greater than that seen in respective WT naive, suggesting a contribution of iNOS to delayed posttraumatic hyperemia. However, pixel analysis denoted that the contribution of iNOS to CBF at $72 \mathrm{~h}$ was not limited to hyperemia flows. In conclusion, iNOS plays a role in the recovery of CBF after CCI in mice. Questions remain if this effect represents a homeostatic component of $\mathrm{CBF}$ recovery, pathologic vasodilatation linked to inflammation, or NO-mediated facilitation of angiogenesis.
\end{abstract}

Key words: arterial spin labeling; head injury; iNOS; magnetic resonance imaging; perfusion

\section{INTRODUCTION}

$\mathbf{I}_{8}$ NDUCIBLE NITRIC OXIDE SYNTHASE (iNOS) has been suggested to play a complex role in the response to central nervous system (CNS) insults such as traumatic brain injury (TBI) and cerebral ischemia. iNOS is induced after injury and is expressed predominantly in resident and infiltrating inflammatory cells and the cerebral vasculature (Clark et al., 1996; Iadecola et al., 1996), although neuronal expression has been reported in some models (Petrov et al., 2000). After experimental TBI in rats, iNOS mRNA expression is detected by $2 \mathrm{~h}$ and peaks at $6 \mathrm{~h}$, while in mice a somewhat more delayed expression between 24 and $72 \mathrm{~h}$ after TBI is observed (Sinz et al.,

\footnotetext{
${ }^{1}$ Pittsburgh NMR Center for Biomedical Research and ${ }^{2}$ Department of Biological Sciences, Carnegie Mellon University, Pittsburgh, Pennsylvania.

${ }^{3}$ Safar Center for Resuscitation Research and ${ }^{4}$ Department of Critical Care Medicine, University of Pittsburgh School of Medicine, Pittsburgh, Pennsylvania.
} 
1999). iNOS has been suggested to produce large quantities of nitric oxide (NO) since- unlike constitutive NOS isoforms - it is not regulated by calcium. Consistent with that notion, iNOS has shown by electron paramagnetic resonance (EPR) spectroscopy to contribute to $\sim 50 \%$ of the NO detected in brain tissue at $72 \mathrm{~h}$ after experimental TBI comparing iNOS knockout (KO) versus wild-type (WT) mice (Bayir et al., 2005).

Overall, most studies suggest that, early after CNS injury, effects of iNOS may be detrimental since treatment with iNOS inhibitors or anti-sense reduces lesion volume, neuronal death, and a number of other markers of acute damage in experimental TBI (Wada et al., 1998a,b), spinal cord injury (Pearse et al., 2003), cryogenic brain injury (Stoffel et al., 2000), and stroke in both rats and mice (Iadecola et al., 1995; Iadecola et al., 1997). However, a dichotomous role for iNOS has been suggested in both experimental TBI and focal cerebral ischemia (Sinz et al., 1999; Bayir et al., 2005; Zhu et al., 2003), and iNOS has been recently shown to mediate ischemic tolerance in mice (Kawano et al., 2007). Several reports have suggested potential beneficial effects of iNOS, particularly at delayed time points after injury. For example, at 17-21 days after TBI, iNOS KO mice exhibit impaired recovery of cognitive outcome on the Morris water maze compared to WT (Sinz et al., 1999). In addition, iNOS KO mice were shown to have greater loss of brain levels of the antioxidant ascorbate versus WT type at 72 $\mathrm{h}$ after experimental TBI (Bayir et al., 2005). Similarly, in experimental stroke, iNOS KO mice exhibited complete absence of the robust neurogenesis response seen in the ipsilateral dentate gyrus at $48 \mathrm{~h}$ in WT (Zhu et al., 2003).

However, despite the known role of $\mathrm{NO}$ as a cerebral vasodilator, studies of the effect of iNOS on CBF after either experimental TBI or ischemia have been limited in contrast to those examining histological, functional, or biochemical endpoints (Willmot et al., 2005), and studies of the role of iNOS in delayed recovery of CBF are lacking. Iadecola et al (1997) reported no acute difference in CBF of the ischemic core or penumbra of iNOS $\mathrm{KO}$ versus WT mice assessed with laser-Doppler flometry during focal cerebral ischemia. To our knowledge, there is only one report on the role of iNOS in the CBF response to experimental TBI. Steiner et al. (2004), using the Marmarou impact acceleration model, reported that rats pre-treated with intracerebroventricular antisense iNOS oligodeoxynucleotides exhibited an exacerbation of the CBF reduction after TBI that was sustained in the initial $48 \mathrm{~h}$ after injury. CBF in that model was also assessed with laser-doppler flometry.

We recently reported the ability to assess regional $\mathrm{CBF}$ in mice-generating maps of $\mathrm{CBF}$ - using the continu- ous arterial spinal labeling (CASL) magnetic resonance imaging (MRI) method (Foley et al., 2005). In the current study, we took advantage of the utility of that approach to quantify maps of regional CBF at 24 and $72 \mathrm{~h}$ after experimental TBI in iNOS KO and WT mice. Our hypothesis was that iNOS would contribute to the level of $\mathrm{CBF}$ at $72 \mathrm{~h}$ after experimental TBI in mice.

\section{METHODS}

\section{Animal Model}

All experiments were approved by the Animal Care and Use Committees of Carnegie Mellon University and the University of Pittsburgh School of Medicine. iNOS $\mathrm{KO}\left(\mathrm{iNOS}^{-/-}\right.$) and WT C57BL/6 (iNOS ${ }^{+/+}$) mice were obtained from Jackson Laboratory (Bar Harbor, ME). The KO mice had been back-bred at least 10 generations. The animals were fed laboratory chow and water $a d l i$ bitum. Mice were maintained under temperature controlled conditions with 12-h light/dark cycles. Knockout mice between the ages of 12 and 15 weeks were assigned into one of three groups: naive $(n=8), 24 \mathrm{~h}(n=8)$, or $72 \mathrm{~h}(n=9)$ after controlled cortical impact (CCI). Wildtype animals were divided into the same groups, naive $(n=9), 24 \mathrm{~h}(n=10)$, and $72 \mathrm{~h}(n=9)$ post CCI.

\section{Controlled Cortical Impact}

A mouse CCI model was used as previously described (Smith et al., 1995) with minor modifications (Whalen et al., 1999). Anesthesia was induced with $3 \%$ isoflurane and anesthesia was maintained with $\mathrm{N}_{2} \mathrm{O} / \mathrm{O}_{2}$ (1:1) and $1-2 \%$ isoflurane via nose cone. Animals were placed in a stereotaxic holder and a temperature probe was inserted through a burr hole into the left frontal cortex and the left parietal bone was removed for trauma. Once brain temperature reached $37^{\circ} \mathrm{C} \pm 0.5^{\circ} \mathrm{C}$ and was maintained at this temperature for $5 \mathrm{~min}$, a vertically directed CCI was delivered (at a velocity of $4.0 \mathrm{~m} / \mathrm{sec}$ and a depth of 1.0 $\mathrm{mm})$. After injury, the bone flap was replaced, sealed with dental cement and the incision closed. Anesthesia was discontinued, and the animals were monitored in supplemental $\mathrm{O}_{2}$ for 30 min and returned to their cages until MRI assessment.

\section{Polymerase Chain Reaction Analysis}

Genotypes of selected iNOS mice were determined by polymerase chain reaction (PCR) according to the protocol set by Jackson Laboratory, the animal provider. Briefly, $15 \mathrm{ng}$ DNA was reacted with $10 \times$ PCR Buffer, $25 \mathrm{mM} \mathrm{MgCl}_{2}, 2.5 \mathrm{mM}$ dNTPs, $5 \mathrm{U} / \mu \mathrm{l}$ Taq Polymerase, $\mathrm{H}_{2} \mathrm{O}$ and $20 \mu \mathrm{M}$ of the three primers designed by the man- 


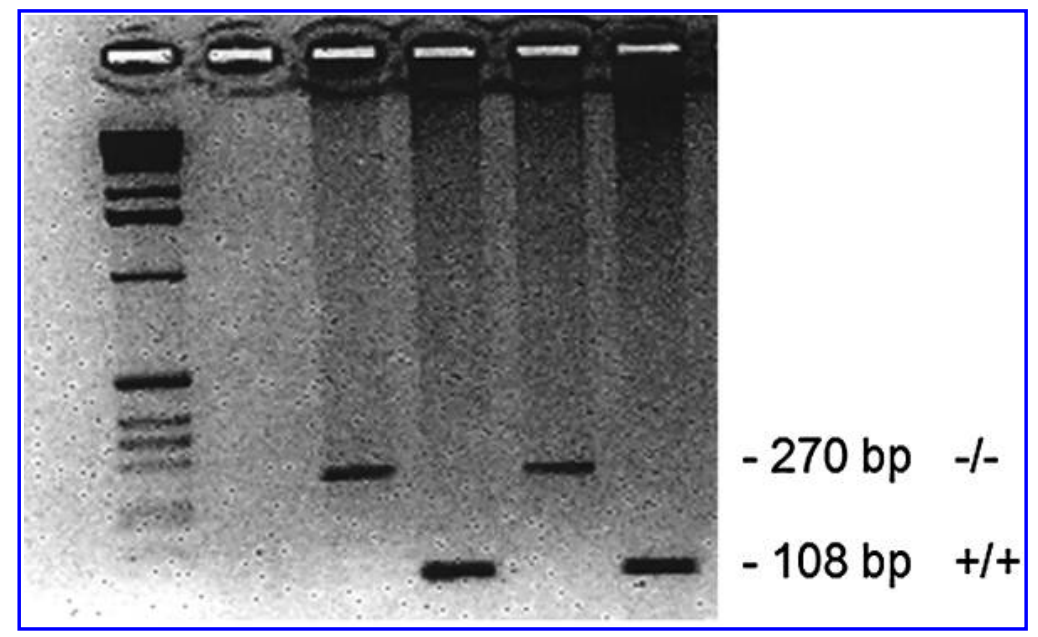

FIG. 1. Polymerase chain reaction (PCR) analysis of inducible nitric oxide synthase (iNOS) genotype. Wild-type mice show a single 108-basepair band, whilst iNOS knock-out mice exhibit a single 270-basepair band.

ufacturer. Cycling reaction times were denaturing at $94^{\circ} \mathrm{C}$ for $1.5 \mathrm{~min}$, annealing at $94^{\circ} \mathrm{C}, 59^{\circ} \mathrm{C}$, and $72^{\circ} \mathrm{C}$, each 30 sec for 35 cycles and extension at $72^{\circ} \mathrm{C}$ for 2 min. PCR products were separated by electorphoresis on a $3 \%$ agarose gel. iNOS WT mice show a single 108-basepair band, while iNOS KO mice exhibit a single 270-basepair band (Fig. 1).

\section{Magnetic Resonance Imaging Protocol}

At the pre-selected time after CCI, anesthesia was induced as previously described (Foley et al., 2005), and mice were intubated and mechanically ventilated with $2 \%$ isoflurane and $\mathrm{N}_{2} \mathrm{O} / \mathrm{O}_{2}(1: 1)$. A femoral artery catheter was surgically inserted for continuous blood pressure monitoring and arterial blood sampling. A rectal temperature probe was inserted for monitoring and maintenance of temperature at $37.0 \pm 0.5^{\circ} \mathrm{C}$ using a warm air heating system (SA Instruments, New York, NY). Mean arterial blood pressure (MABP) and heart rate were also monitored throughout image acquisition. Arterial blood gases were collected at the beginning and end of the studies; ventilator rate and tidal volume were adjusted to maintain arterial $\mathrm{CO}_{2}$ tension $\left(\mathrm{PaCO}_{2}\right)$ of $30-45 \mathrm{~mm} \mathrm{Hg}$. Studies were discarded if the final $\mathrm{PaCO}_{2}$ measurement was outside this range. Mice were placed onto a cradle in the prone position, and the head was secured with ear bars and an adjustable bite bar to limit motion.

\section{Magnetic Resonance Image Acquisition}

MR studies were performed on a 4.7-Tesla, 40-cmbore Bruker AVANCE system, equipped with a 15-cmdiameter shielded gradient insert and a home-built saddle-type RF coil. Image acquisition parameters were identical to those reported in a prior study (Foley et al., 2005). Briefly, $T_{2}$-weighted spin-echo images were used to verify the position of the center of the contusion, and were acquired with the following parameters: field of view $2.5 \mathrm{~cm}, 1$-mm-slice thickness, 1.5-mm-interslice distance, $T R / T E=2500 / 40 \mathrm{msec}$, two averages, five slices, and a $128 \times 70$ matrix interpolated to $128 \times 128$. Perfusion studies were performed using a continuous arterial spin labeling (Detre et al., 1992; Williams et al., 1999), imaging technique (spin echo, $64 \times 40$ matrix interpolated to $64 \times 64, T R=2000 \mathrm{msec}$, summation of three echoes, $T E=10,20$, and $30 \mathrm{msec}$, and two averages). The labeling pulse for the inversion plane was positioned $\pm 2 \mathrm{~cm}$ from the perfusion detection plane. Spinlabeling efficiency (Zhang et al., 1993) was determined from intensities within the carotid arteries (gradient echo, $45^{\circ}$ flip angle, eight averages, TR/TE $=100 / 9.6 \mathrm{msec}$, $256 \times 256$ matrix, and spin-labeling applied at $\pm 6 \mathrm{~mm})$. The spin-lattice relaxation time of tissue water ( $\left.T_{1 \mathrm{obs}}\right)$ (Hendrich et al., 1999) was measured from a series of spin-echo images $(T R=8000,4300,2300,1200,650$, 350,185 , and $100 \mathrm{msec}, T E=9 \mathrm{msec}$, two averages, and a $64 \times 40$ matrix interpolated to $64 \times 64)$.

\section{Image Analysis}

All image processing was performed with the Bruker ParaVision 3.0.2 image analysis software. Regions of interest (ROIs) defined the left (ipsilateral) and right (contralateral) hemisphere. Regions within each hemisphere including the cortex, hippocampus, thalamus and the amygdala/piriform cortex were also defined, guided by assignments from a mouse brain atlas (Sidman et al., 1971). Cortical ROIs were drawn to include the entire 
FOLEY ET AL.

Table 1. Physiological Data ${ }^{a}$

\begin{tabular}{lcccccc}
\hline & $\begin{array}{c}\text { WT naive } \\
(\mathrm{n}=9)\end{array}$ & $\begin{array}{c}\text { KO naive } \\
(\mathrm{n}=8)\end{array}$ & $\begin{array}{c}\text { WT 24- } \text { h post-CCI } \\
(\mathrm{n}=10)\end{array}$ & $\begin{array}{c}\text { KO 24 h post-CCI } \\
(\mathrm{n}=8)\end{array}$ & $\begin{array}{c}\text { WT } 72-\text { h post-CCI } \\
(\mathrm{n}=9)\end{array}$ & $\begin{array}{c}\text { KO } \\
\text { 72-h post-CCI } \\
(\mathrm{n}=9)\end{array}$ \\
\hline $\mathrm{MABP}^{\mathrm{b}}$ & $74.9 \pm 15.4$ & $91.9 \pm 8.6^{\#}$ & $83.2 \pm 8.0$ & $80.8 \pm 16.0$ & $91.5 \pm 9.9$ & $91.2 \pm 11.6$ \\
$\mathrm{PaCO}_{2}$ & $38.3 \pm 7.2$ & $35.9 \pm 4.5$ & $44.2 \pm 14.2$ & $36.9 \pm 5.0$ & $36.4 \pm 3.0$ & $36.0 \pm 6.1$ \\
$\mathrm{PaO}_{2}$ & $229.7 \pm 58.0$ & $244.0 \pm 44.0$ & $212.8 \pm 51.4$ & $238.7 \pm 22.7$ & $249.3 \pm 51.4$ & $225.0 \pm 50.5$ \\
$\mathrm{pH}$ & $7.28 \pm 0.03$ & $7.36 \pm 0.05^{*}$ & $7.25 \pm 0.09$ & $7.33 \pm 0.05^{*}$ & $7.28 \pm 0.06$ & $7.36 \pm 0.05^{*}$ \\
Hematocrit & $56.0 \pm 10.0$ & $49.0 \pm 9.0$ & $48.0 \pm 7.0$ & $44.0 \pm 13.0$ & $48.0 \pm 9.0$ & $43.0 \pm 6.0$ \\
Heart rate & $435.0 \pm 152.0$ & $452.0 \pm 71.0$ & $404.0 \pm 128.0$ & $596.0 \pm 86.0$ & $431.0 \pm 123.0$ & $478.0 \pm 102.0$ \\
Weight $(\mathrm{g})$ & $26.0 \pm 2.0$ & $27.0 \pm 3.0$ & $26.0 \pm 2.0$ & $28.0 \pm 1.0 *$ & $26.0 \pm 2.0$ & $27.0 \pm 3.0$ \\
\hline
\end{tabular}

${ }^{\text {aD }}$ ata are mean $\pm \mathrm{SD}$.

${ }^{\mathrm{b}}$ At time of perfusion experiment.

$* p<0.05$ versus naive.

${ }^{\#} p<0.05$ versus WT.

WT, wild-type; KO, iNOS knockout; CCI, controlled cortical impact; MABP, mean arterial blood pressure.

cortex within each hemisphere (cortex), or an area beginning $1 \mathrm{~mm}$ from midline to the midpoint of the arc of the cortex (cortex-CR). The cortex-CR region, after CCI, contains an area that has a large contribution from the contusion. Pixel by pixel maps of $\left(M_{\mathrm{C}}-M_{\mathrm{L}}\right) \cdot M_{\mathrm{C}}{ }^{-1}$ were generated from the perfusion data. $M_{\mathrm{C}}$ is the magnetization intensity from the control image and $M_{\mathrm{L}}$ is the magnetization intensity from the labeled image. The average of the two maps was computed and negative pixels in the brain were retained for quantification. $T_{1 \mathrm{obs}}$ maps were generated from the series of variable $T R$ images by a three parameter non-linear fit to:

$$
M(T R)=M_{0}\left[1-A \exp \left(-T R / T_{1 \mathrm{obs}}\right)\right],
$$

where $M(T R)$ is the signal intensity for each $T R$ value, $M_{0}$ is the signal intensity at equilibrium, and $A$ is the saturation correction factor. A mean value of the perfusion maps and $T_{1 \text { obs }}$ were computed for each ROI. Regional CBF was then calculated from (Zhang et al., 1995):

$$
\mathrm{CBF}=\lambda \cdot\left(T_{1 \mathrm{obs}} \cdot 2 \alpha\right)^{-1} \cdot\left(M_{\mathrm{C}}-M_{\mathrm{L}}\right) \cdot M_{\mathrm{C}}{ }^{-1}
$$

where $\lambda$ is the blood-brain partition coefficient of water, with a spatially constant value of $0.9 \mathrm{~mL} / \mathrm{g}$ assumed (Herscovitch and Raichle, 1985), and $\alpha$ is the spin-labeling efficiency measured in the carotids. Pixel analysis was performed using ImageJ software (Abramoff et al., 2004). ROI's were segmented from each CBF map and histograms were generated for each timepoint and geneotype.

\section{Statistical Analysis}

All data are expressed as mean \pm standard deviation. Independent iNOS KO and WT mice were studied for each time point (naive, $24 \mathrm{~h}$, and $72 \mathrm{~h}$ ) and thus all phys- iological data from KO and WT were compared at each time point using either a two-tailed Student's $t$ test or analysis of variance (ANOVA). A probability of less than $0.05(p<0.05)$ was considered significant.

\section{RESULTS}

\section{Physiology}

Temperature at the time of $\mathrm{CBF}$ determination was maintained at $37.0^{\circ} \mathrm{C} \pm 0.5^{\circ} \mathrm{C}$ for all mice. Arterial blood gases were measured at both the beginning and conclusion of each MRI experiment and averaged. Mean $\mathrm{PaCO}_{2}$ and $\mathrm{PO}_{2}$ values did not significantly differ between WT and $\mathrm{KO}$ mice or between injured and uninjured animals (Table 1). MABP at the time of CBF determination was $74.9 \pm 15.4, \quad 91.9 \pm 8.6, \quad 83.2 \pm 8.0, \quad 80.8 \pm 16.0$, $91.5 \pm 9.9,91.2 \pm 11.6 \mathrm{~mm} \mathrm{Hg}$ for the WT naive, KO naive, WT $24 \mathrm{~h}$ post-CCI, KO $24 \mathrm{~h}$ post-CCI, WT $72 \mathrm{~h}$ post-CCI, and KO $72 \mathrm{~h}$ post-CCI groups, respectively (Table 1). MABP for the KO naive mouse was significantly higher than that of the WT naive, but all MABP readings were in a physiologically acceptable range. Hematocrits did not differ between groups (Table 1). The average weight of the animals used in the KO $24 \mathrm{~h}$ postCCI group was slightly, albeit significantly higher than WT. The mean $\mathrm{pH}$ of the iNOS KO groups was modestly, albeit significantly higher than that of the WT mice, but all were in a physiologically acceptable range.

$T_{\text {lobs }}$ Maps, Spin Labeling Efficiency $(\alpha)$, and Regional Cerebral Blood Flow

Mean $T_{1 \text { obs }}$ values for all studies are listed in Table 1. After CCI, $T_{1 \text { obs }}$ values ipsilateral to the injury were sig- 
INDUCIBLE NITRIC OXIDE SYNTHASE AND CEREBRAL BLOOD FLOW AFTER TBI

Table 2. Regional $T_{10 b s}$ (Sec) and Arterial Spin Labeling Efficiency $(\alpha)^{\mathrm{a}}$

\begin{tabular}{|c|c|c|c|c|c|c|}
\hline & $\begin{array}{c}\text { WT naive } \\
(\mathrm{n}=9)\end{array}$ & $\begin{array}{c}\text { KO naive } \\
(\mathrm{n}=8)\end{array}$ & $\begin{array}{l}W T 24-h \\
\text { post-CCI } \\
(\mathrm{n}=10)\end{array}$ & $\begin{array}{c}\text { KO } 24 h \\
\text { post-CCI } \\
(\mathrm{n}=8)\end{array}$ & $\begin{array}{c}W T 72-h \\
\text { post-CCI } \\
(\mathrm{n}=9)\end{array}$ & $\begin{array}{c}\text { KO } 72-h \\
\text { post-CCI } \\
(\mathrm{n}=9)\end{array}$ \\
\hline \multicolumn{7}{|l|}{$T_{1 \mathrm{obs}}$} \\
\hline \multicolumn{7}{|c|}{ Hemisphere } \\
\hline Left & $1.62 \pm 0.08$ & $1.64 \pm 0.07$ & $2.01 \pm 0.24^{*}$ & $2.09 \pm 0.33^{*}$ & $2.11 \pm 0.62 *$ & $1.78 \pm 0.16^{*}$ \\
\hline Right & $1.63 \pm 0.10$ & $1.67 \pm 0.05$ & $1.71 \pm 0.12$ & $1.69 \pm 0.05$ & $1.74 \pm 0.16$ & $1.63 \pm 0.11$ \\
\hline \multicolumn{7}{|l|}{ Cortex } \\
\hline Left & $1.66 \pm 0.09$ & $1.67 \pm 0.07$ & $2.54 \pm 0.88^{*}$ & $2.68 \pm 0.75^{*}$ & $2.66 \pm 1.30 *$ & $2.10 \pm 0.32 *$ \\
\hline Right & $1.69 \pm 0.12$ & $1.69 \pm 0.06$ & $1.78 \pm 0.18$ & $1.76 \pm 0.10$ & $1.84 \pm 0.31$ & $1.70 \pm 0.12$ \\
\hline \multicolumn{7}{|c|}{ Cortex-CR } \\
\hline Left & $1.64 \pm 0.09$ & $1.66 \pm 0.06$ & $3.00 \pm 1.59 *$ & $3.26 \pm 1.86^{*}$ & $3.66 \pm 2.67 *$ & $2.23 \pm 0.47 *$ \\
\hline Right & $1.66 \pm 0.13$ & $1.68 \pm 0.07$ & $1.72 \pm 0.14$ & $1.76 \pm 0.15$ & $1.88 \pm 0.44$ & $1.66 \pm 0.15$ \\
\hline \multicolumn{7}{|c|}{ Thalamus } \\
\hline Left & $1.44 \pm 0.06$ & $1.50 \pm 0.06$ & $1.56 \pm 0.10^{*}$ & $1.46 \pm 0.04^{\#}$ & $1.48 \pm 0.12$ & $1.41 \pm 0.08^{\#}$ \\
\hline Right & $1.45 \pm 0.08$ & $1.53 \pm 0.08^{\#}$ & $1.54 \pm 0.11$ & $1.50 \pm 0.06$ & $1.51 \pm 0.14$ & $1.46 \pm 0.11$ \\
\hline \multicolumn{7}{|c|}{ Amygdala/piriform cortex } \\
\hline Left & $1.80 \pm 0.16$ & $1.78 \pm 0.14$ & $1.85 \pm 0.19$ & $1.73 \pm 0.05$ & $1.79 \pm 0.21$ & $1.69 \pm 0.11$ \\
\hline Right & $1.80 \pm 0.14$ & $1.82 \pm 0.14$ & $1.78 \pm 0.03$ & $1.77 \pm 0.06$ & $1.80 \pm 0.24$ & $1.74 \pm 0.17$ \\
\hline \multicolumn{7}{|c|}{ Hippocampus } \\
\hline Left & $1.69 \pm 0.15$ & $1.69 \pm 0.07$ & $1.99 \pm 0.23^{*}$ & $1.95 \pm 0.29 *$ & $1.73 \pm 0.16$ & $1.76 \pm 0.23$ \\
\hline Right & $1.74 \pm 0.17$ & $1.79 \pm 0.16$ & $1.80 \pm 0.17$ & $1.87 \pm 0.16$ & $1.78 \pm 0.20$ & $1.70 \pm 0.19$ \\
\hline$\alpha$ & $0.68 \pm 0.04$ & $0.70 \pm 0.05$ & $0.69 \pm 0.04$ & $0.67 \pm 0.03$ & $0.67 \pm 0.04$ & $0.68 \pm 0.05$ \\
\hline
\end{tabular}

${ }^{a}$ Data are mean \pm SD.

$* p<0.05$ versus naive.

${ }^{\#} p<0.05$ versus WT.

WT, wild-type; KO, iNOS knockout; CCI, controlled cortical impact.

nificantly elevated. The $T_{1 \mathrm{obs}}$ of the contusion rich area of the cortex was $3.10 \pm 1.59 \mathrm{sec}$ on the injured side versus $1.72 \pm 0.13 \mathrm{sec}$ on the contralateral side for WT mice $24 \mathrm{~h}$ post-CCI, the KO animals had similar values (Table 2). $T_{1 \text { obs }}$ was $1.64 \pm 0.09$ and $1.66 \pm 0.13 \mathrm{sec}$ in the ipsilateral and contralateral contusion rich area of the cortex of the WT mice, again the KO mice were similar (Table 2). $T_{1 \text { obs }}$ values were also significantly increased in the ipsilateral hemisphere, cortex and hippocampus of WT and KO mice $24 \mathrm{~h}$ post-CCI and the ipsilateral hemisphere, cortex and cortex-CR of WT and KO mice $72 \mathrm{~h}$ post-CCI (Table 2). The mean values for $\alpha$ were 0.68 , $0.7,0.69,0.67,0.67$, and 0.68 for the naive WT and $\mathrm{KO}$, WT and KO $24 \mathrm{~h}$ post-CCI, and WT and KO $72 \mathrm{~h}$ postCCI groups, respectively, and did not statistically differ between groups (Table 2).

Representative CBF maps obtained from naive mice and after CCI are shown in Figure 2. Regions with low perfusion generally corresponded to areas with elevated

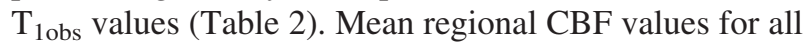
studies are shown in Figure 3. Comparing anatomical brain ROIs at $24 \mathrm{~h}$ post-CCI, there were significant reductions in $\mathrm{CBF}$ in the injured animals versus naive, re- gardless of genotype. Values for the ipsilateral hemisphere of WT animals at $24 \mathrm{~h}$ post-CCI were reduced by $31 \%$, while the cortex and cortex-CR flows were reduced by $57 \%$ and $76 \%$, respectively, compared to naives. $\mathrm{KO}$ mice at $24 \mathrm{~h}$ after CCI experienced similar decreases in $\mathrm{CBF}$ as the WT of $38 \%, 67 \%$, and $85 \%$ in the hemisphere, cortex and cortex-CR, respectively; however, the $\mathrm{KO}$ mice also exhibited a significant reduction of $\mathrm{CBF}$ in the hippocampus versus respective naive (Fig. 3). In contrast, regional assessment of $\mathrm{CBF}$ at $72 \mathrm{~h}$ after injury demonstrated that recovery of $\mathrm{CBF}$ was reduced in the ipsilateral hippocampus, thalamus, and amygdala/piriform cortex in iNOS KO versus WT mice by $26 \%, 15 \%$, and $21 \%$ respectively. Of note, these three regions with reduced $\mathrm{CBF}$ in iNOS KO versus WT at $72 \mathrm{~h}$ represented ROIs where CBF in the WT was either numerically or statistically greater than that seen in respective WT naive - suggesting posttraumatic hyperemia. The attenuated recovery of CBF in iNOS KO versus WT at $72 \mathrm{~h}$ after CCI was restricted to these three ROIs since $\mathrm{CBF}$ in iNOS KO and WT mice at $72 \mathrm{~h}$ post-CCI in the cortex and cortex-CR regions did not differ between genotypes and remained significantly lower than naives (Fig. 3). 


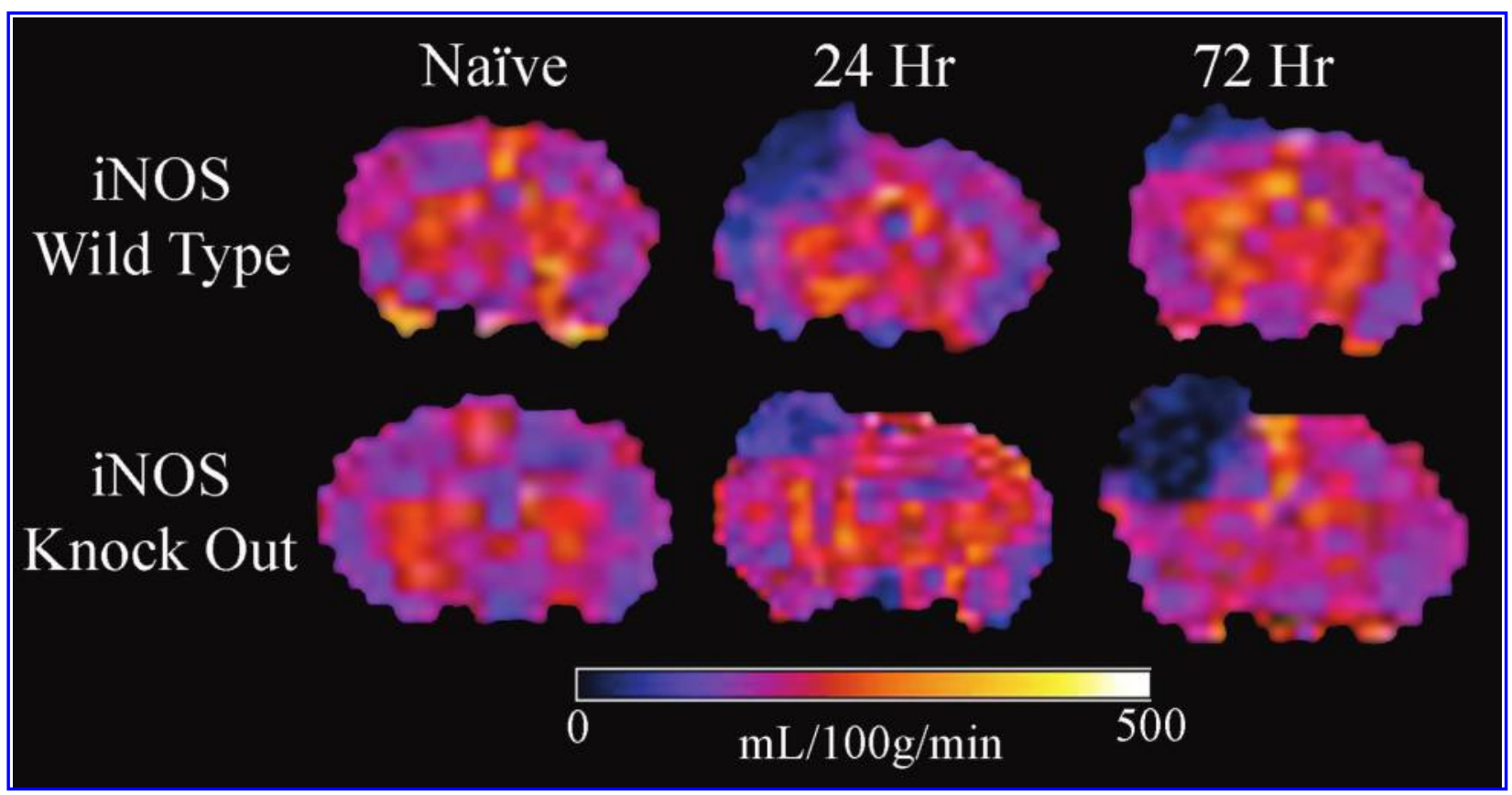

FIG. 2. Representative cerebral blood flow (CBF) maps of wild-type (WT) and inducible nitric oxide synthase (iNOS) knockout (KO) mice without injury (naive) and at $24 \mathrm{~h}$ and $72 \mathrm{~h}$ after controlled cortical impact (CCI) injury. There was no difference in $\mathrm{CBF}$ in any region of interest (ROI) in naive WT versus iNOS KO mice. After injury, maps show spatial heterogeneity. At 24 $\mathrm{h}$ after injury, a region of reduced CBF is readily identified in the contused cortex of both WT and iNOS KO mice. Similarly, at $24 \mathrm{~h}$ after CCI, overall hemispheric CBF was reduced ipsilateral to injury in both WT and iNOS KO mice. At $72 \mathrm{~h}$ after injury, recovery of $\mathrm{CBF}$ was attenuated in iNOS KO versus WT. Maps represent the best example of the average CBF for each group. Pixels outside the brain have been assigned zero intensity.

To gain additional insight of the effect of iNOS in these different brain regions we examined the distribution of blood flow in selected brain regions in the WT versus the KO. Pixel analysis of CBF maps from all animals at 72 $\mathrm{h}$ show that for the contralateral side there is no obvious difference between genotype (Fig. 4A). The most interesting finding came when the ipsilateral hippocampus was analyzed (Fig. 4B). Pixels with a CBF below 100 $\mathrm{mL} / 100 \mathrm{~g} / \mathrm{min}$ were only seen in the iNOS KO mice. This is consistent with the notion that CBF recovery is blunted in the ipsilateral hippocampus, and that this effect is not limited to the attenuation of hyperemic flows.

\section{DISCUSSION}

Our data suggest that iNOS plays a role in the normal recovery of $\mathrm{CBF}$ after $\mathrm{CCI}$ in mice. iNOS expression may contribute as much as $26 \%$ to the observed CBF following injury and also depends on the time after injury and brain region. Attenuated recovery of CBF in iNOS KO versus WT occurred predominantly at $72 \mathrm{~h}$ after injury and was restricted to structures outside of the contusion.
Curiously, those regions generate supra-normal CBF values in WT at $72 \mathrm{~h}$ after injury.

After TBI in rats using a weight-drop model iNOS positive cells are seen around the injury site at 4-6 h, expression peaks at $\sim 24 \mathrm{~h}$, and gradually decreases (Gahm et al., 2000). In immature rats using a weight-drop model, iNOS expression was noted at low levels as soon as $2 \mathrm{~h}$ after injury but marked at 24 and $48 \mathrm{~h}$ (Clark et al., 1996). Wada et al. (1998a,b) saw increased iNOS activity at 3 days after fluid-percussion injury with the most dramatic increase occurring at 7 days in adult rats. The predominant cell types expressing iNOS are neutrophils (Clark et al., 1996; Gahm et al., 2000), macrophages (Gahm et al., 2000; Petrov et al., 2000) and vascular smooth muscle cells, which suggests that iNOS may play a role in cerebrovasodilation (Clarke et al., 1996). Petrov et al. (2000) found that $24 \mathrm{~h}$ after TBI, using a weight drop model in rats, there is also iNOS expression in injured neurons. It is interesting to note that in the naive animals there was no difference in CBF between WT and KO. This is consistent with the notion that iNOS is induced and not present in significant quantities in the normal brain.

It is not clear if the effect of iNOS on CBF at $72 \mathrm{~h}$ af- 


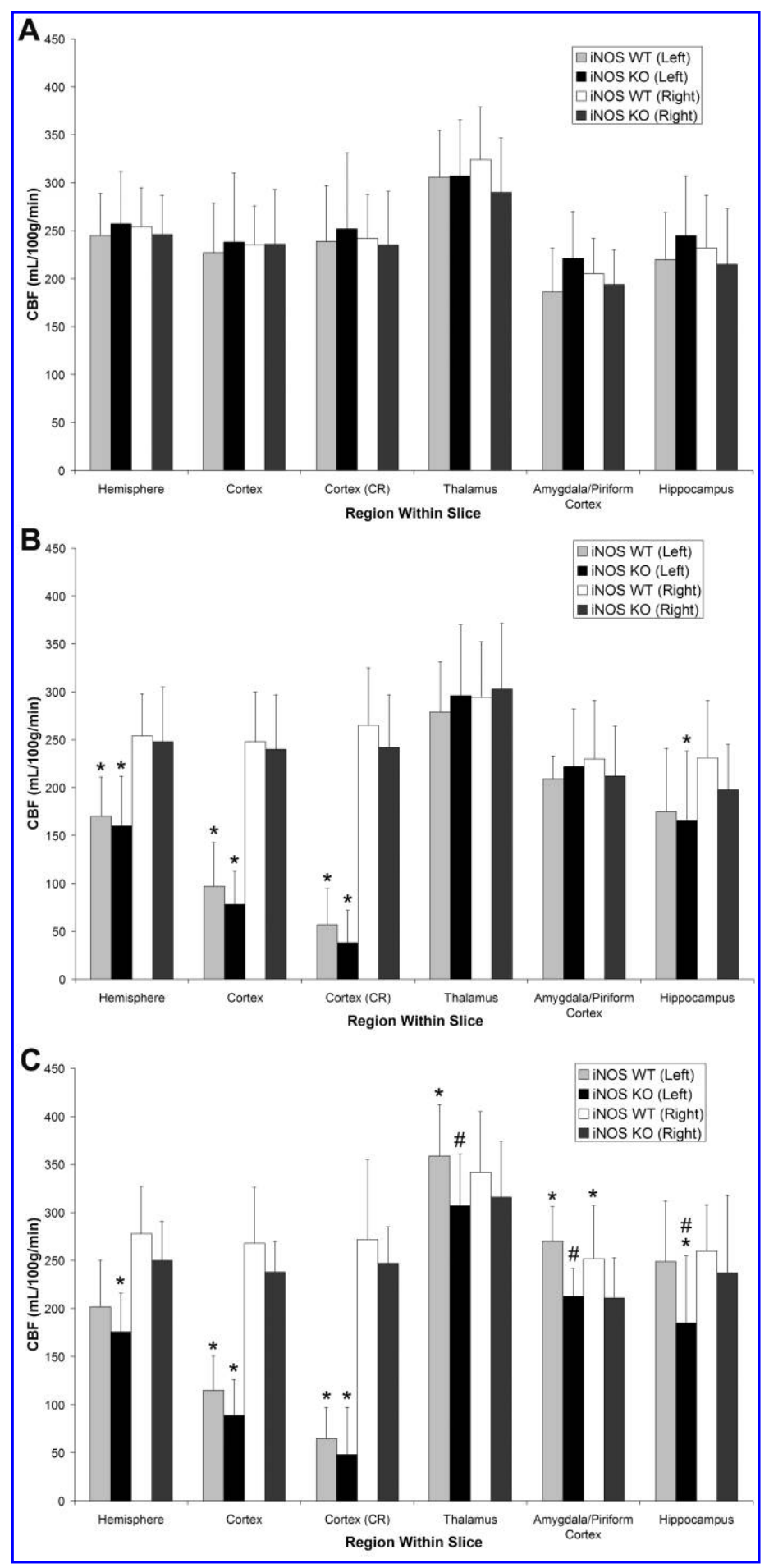

FIG. 3. Regional cerebral blood flow (CBF) values (mean \pm SD) from studies with naive wild-type (WT; $n=9)$ and knockout $(\mathrm{KO} ; n=8)$ mice $(\mathbf{A})$, WT $24 \mathrm{~h}(n=10)$, and KO $24 \mathrm{~h}(n=8)$ after controlled cortical impact $(\mathrm{CCI})(\mathbf{B})$, and WT $72 \mathrm{~h}(n=$ 9) and KO $72 \mathrm{~h}(n=9)$ post-CCI $(\mathbf{C})$. Standard deviation (SD) in CBF represents the intra-group variability computed from $\left(M_{\mathrm{C}}-M_{\mathrm{L}}\right) \cdot M_{\mathrm{C}}{ }^{-1} .{ }^{*} p<0.05$ versus naive and ${ }^{\#} p<0.05$ versus WT by Student's $t$-test. 


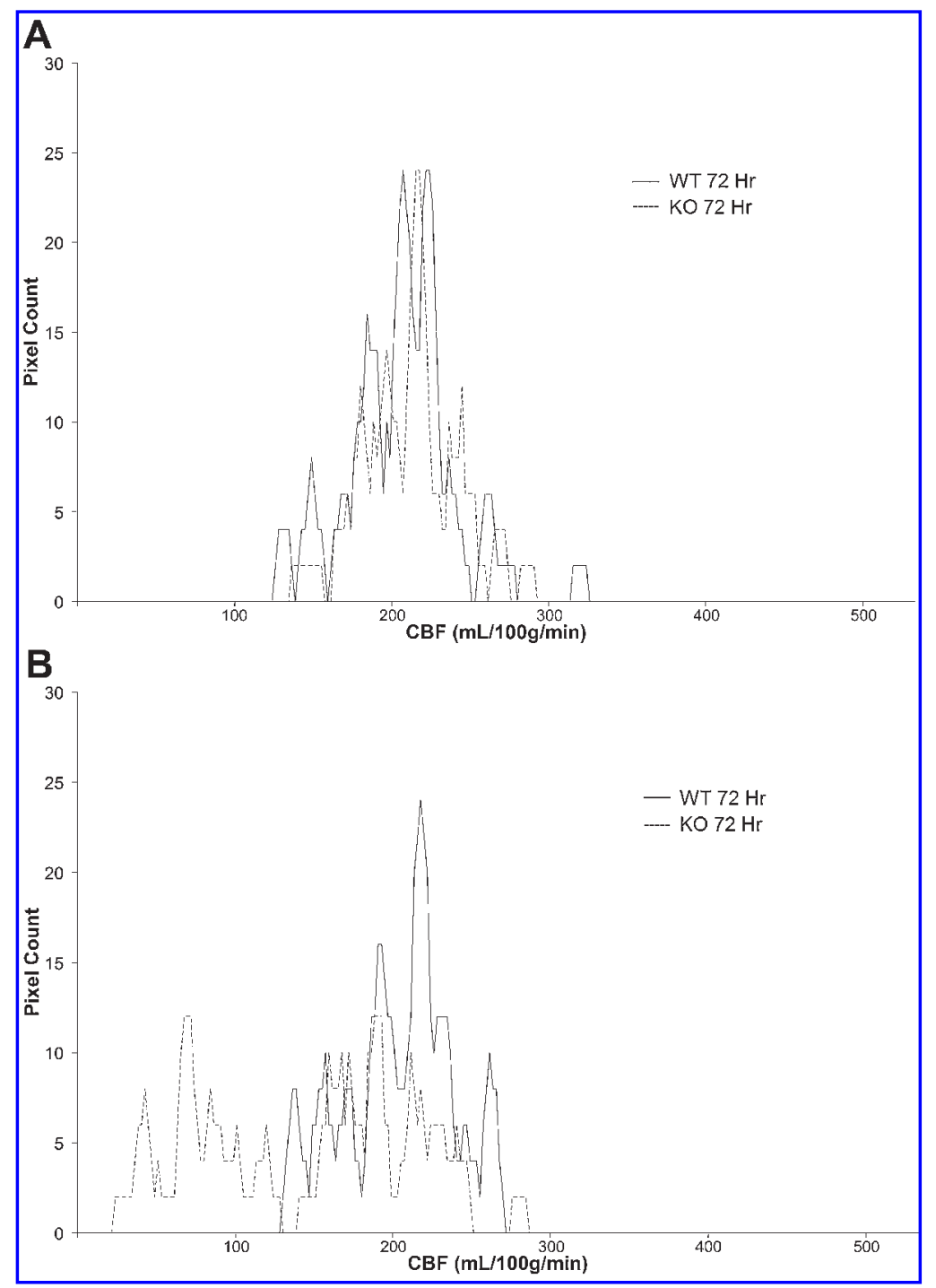

FIG. 4. Pixel analysis of cerebral blood flow (CBF) maps for all inducible nitric oxide synthase (iNOS) wild-type (WT) and knockout (KO) mice at $72 \mathrm{~h}$ after experimental traumatic brain injury (TBI). Contralateral (A) and ipsilateral (B) hippocampus.

ter injury in our model represents enhanced recovery or a pathological process. In this same mouse model, we previously reported that the iNOS $\mathrm{KO}$ exhibited poorer functional recovery than the WT between 17 and 21 days after injury, as assessed by Morris water maze performance (Sinz et al., 1999), but no significant difference between genotypes was noted in contusion volume at 21 days. Conversely, administration of the iNOS inhibitor aminoguanidine (AG) reduced the number of necrotic neurons seen after fluid percussion injury in rats, but did not reduce contusion volume (Wada et al., 1998a,b). The delayed use of the more selective iNOS inhibitor 1400W, given at 18 or $24 \mathrm{~h}$ after injury, improved histopathological outcome (Jafarian-Tehrani et al., 2005). Louin et al. (2006) investigated the effect of three different iNOS inhibitors, each with increasing selectivity-AG, L- $N$ iminoethyl-lysine (L-NIL), and 1400W-after TBI and showed that each inhibitor improved neurological function at $24 \mathrm{~h}$ (Louin et al., 2006). However, sustained treatment with AG for 5 days or L -NIL for 1.5 days worsened histological outcome at 21 days after CCI in rats (Sinz et al., 1999). Taken together, iNOS appears to mediate detrimental effects early — in the initial $24 \mathrm{~h}$ - while mediating beneficial actions at more delayed time points. The observed deficit in CBF recovery at $72 \mathrm{~h}$ but not 24 $h$ after TBI in the setting of iNOS deficiency in our study is consistent with a more dominant role for iNOS in the recovery phase, rather than the acute phase. Given that iNOS induction after CCI in mice is a fairly delayed event, and that many early events in the secondary injury 
cascade, such as acute energy failure, play an important role in tissue loss, it is reasonable that increased contusion volume was not observed in the iNOS KO versus WT.

Using EPR spectroscopy, we previously reported (Bayir et al., 2005) that the NO levels are $\sim 50 \%$ lower in the injured hemisphere at $72 \mathrm{~h}$ after CCI in iNOS KO versus WT mice in this exact model. Thus, loss of a direct effect of $\mathrm{NO}$ in iNOS KO mice likely explains our findings. There are several possibilities to consider. Induction of iNOS and production of iNOS-derived NO in the regions outside of the contusion may mediate (1) direct vasodilatory effects in the delayed recovery of $\mathrm{CBF}$, or (2) pathological hyperemic $\mathrm{CBF}$ as part of the inflammatory process in brain, and/or angiogenesis in brain regions recovering and/or remodeling after CCI.

Rats pre-treated with anti-sense iNOS oligodeoxynucleotides exhibited an exacerbation of the CBF reduction after TBI, produced by impact acceleration, which was sustained in the initial $48 \mathrm{~h}$ (Steiner et al., 2004). An interaction between iNOS and endothelin-1 (ET-1) was suggested to mediate this $\mathrm{CBF}$ reduction in those studies since the posttraumatic increases in ET-1 levels were augmented between 4 and $48 \mathrm{~h}$ after injury. A synergistic loss of vasodilatory effects of NO and enhanced expression of ET-1 in iNOS KO mice was proposed to mediate this $\mathrm{CBF}$ effect, since NO exhibits an inhibitory influence on activator protein-1 (AP-1) — a known up-regulator of ET-1 expression. However, the work by Steiner et al (2004) had several limitations including the use of laser-Doppler flow assessment, and thus provided only a relative flow assessment. There was also no quantification of relative flow in that study or statistical comparison between groups, in lieu of presentation of the slope of the regression line of mice in each group over timemaking it difficult to appreciate the magnitude of the effect of inhibition of iNOS on CBF. Nevertheless, a contribution of iNOS to ameliorating posttraumatic hypoperfusion was supported. We did not assess ET-1 levels, and thus cannot comment on possible synergism between loss of NO and increases in ET-1 in our model.

In our study, the effects of genotype on CBF were largely restricted to blunting of delayed posttraumatic increases in CBF normally observed in subcortical ROIs such as thalamus and amygdala. iNOS has been shown to mediate cerebral hyperemia in the inflammatory response, such as that observed in rat brain in response to endotoxin administration (Okamoto et al., 1998). There is robust accumulation of local and infiltrating inflammatory cells after injury in the mouse CCI model supporting this possibility (Carlos et al., 1997; Bayir et al., 2005). Given the severity of the effect of CCI on the contused cortex, it would also be reasonable to speculate that the lack of effect of genotype on CBF within the contusion represents lack of responsivity of the severely damaged vasculature to NO in that region. Finally, our pixel analysis in hippocampus revealed that the effect of iNOS on $\mathrm{CBF}$ was not limited to a reduction in hyperemic flows-given that pixels with $\mathrm{CBF}$ values of $<100$ $\mathrm{mL} / 100 \mathrm{~g} / \mathrm{min}$ were seen only in the iNOS KO. Indeed, pixels with $\mathrm{CBF}$ values of $<50 \mathrm{~mL} / 100 \mathrm{~g} / \mathrm{min}$ were seen. It is not clear, however, whether this finding is mediating, in part, the impaired water maze performance previously reported after CCI in this genotype (Sinz et al., 1999). Indeed, the implications of the effect of iNOS on CBF recovery after CCI remain to be defined.

Over-expression of iNOS was recently shown to induce angiogenesis and regulate the degree of perfusion in tumors (Cullis et al., 2006). Similarly, a critical role for iNOS in neurogenesis after focal cerebral ischemia is known (Zhu et al., 2003). These effects may be mediated by cGMP as suggested by Zhang et al. (2002). Given the rapid time course that has been reported for angiogenesis in experimental CNS injury, where a significant increase in the number of vessels can be seen in post-ischemic brain at 3 days (Hayashi et al., 2003), we cannot rule out the possibility that the attenuated $\mathrm{CBF}$ response seen in iNOS $\mathrm{KO}$ mice results from the preclusion of iNOS-mediated angiogenesis.

Whether iNOS-mediated effects on CBF after TBI are beneficial, detrimental, or and epiphenomenon remains unclear. Cerebral metabolic rate, as assessed by deoxyglucose utilization, at 24 or $72 \mathrm{~h}$ after CCI in rodents is generally reduced (Hovda et al., 1995), suggesting that hyperemic flows at $72 \mathrm{~h}$ after injury may be pathologic. However, a regional assessment of cerebral metabolic rate was not carried out in this study. Similarly, the specific cell types mediating the effects of iNOS on CBF after TBI remain to be determined. These represent logical areas for future investigation.

There are limitations to this work. Arterial $\mathrm{pH}$ was slightly lower in WT versus KO mice for each comparison-naive, $24 \mathrm{~h}$, and $72 \mathrm{~h}$. We do not anticipate that this difference would importantly influence $\mathrm{CBF}$ since $\mathrm{PaCO}_{2}$ was controlled and did not differ between groups. This suggests that the difference was related to a mild metabolic acidosis in WT that was blunted in the KO. The reason for this finding remains unclear; however, systemic effects of loss of iNOS-derived NO cannot be ruled out. MABP was also higher in the naive $\mathrm{KO}$, which was a surprising finding. However, all mice had a MABP in the physiologically acceptable range and no difference in MABP was seen between genotypes after TBI. Second, because of the need for invasive blood gases and blood pressure monitoring, it was not possible to serially study mice-despite the fact that the CASL method af- 
fords the advantage of being a non-invasive approach. Assessing the temporal profile of CBF across time in individual mice would have been desirable. Finally, we did not measure NO levels in these mice to confirm an effect of iNOS on NO production in brain; however, we previously reported a $\sim 50 \%$ reduction in NO levels by EPR spectroscopy in the injured hemisphere in this model in iNOS KO versus WT mice (Bayir et al., 2005).

Anesthetic preconditioning with isoflurane is recognized to be neuroprotective. Pretreatment with isoflurane for a period of $30 \mathrm{~min}$ to $3 \mathrm{~h}$ induces a time-dependent increase in iNOS protein expression. Inhibition of iNOS abolished the isoflurane preconditioning induced neuroprotection (Kapinya et al., 2002; Zhao and Zuo, 2004). Although we used isoflurane anesthesia in our studies, at $24 \mathrm{~h}$ post-CCI when iNOS expression from isoflurane induction is reported to be at a peak (Kapinya et al., 2002), there was no important difference in CBF between iNOS WT and KO mice. This suggests that it is unlikely that isoflurane played a key role in our findings. However, studies using additional anesthetics are warranted.

In conclusion, iNOS plays a role in the recovery of CBF after CCI in mice. Attenuated recovery of CBF in iNOS $\mathrm{KO}$ versus WT predominantly occurred at $72 \mathrm{~h}$ after injury and was restricted to structures outside of the contusion. Whether this effect of iNOS represents a homeostatic component of CBF recovery, pathologic vasodilatation linked to inflammation, or results from NO-mediated facilitation of angiogenesis remains to be determined.

\section{ACKNOWLEDGMENTS}

We thank Keri Feldman for technical assistance with genotyping and Marci Provins for secretarial assistance with preparation of the manuscript. We thank NINDSNS 30318 (to P.M.K.) and NIBIB P41 EB-001977 (to C.H.) for support. The Pittsburgh NMR Center for Biomedical Research is supported by a grant from the National Institute of Biomedical Imaging and Bioengineering as an NIH-supported Resource Center (P41EB001977).

\section{DISCLOSURE STATEMENT}

No competing financial interests exist. Patent holder on Emergency Preservation and Resuscitation Method.

\section{REFERENCES}

Abramoff, M.D., Magelhaes, P.J., and Ram, S.J. (2005). Image processing with Image J. Biophotonics Int. 11, 36-42.
Bayir, H., Kagan, V.E., Borisenko, G.G., Tyurina, Y.Y., Janesko K.L., Vagni, V.A., Billiar, T.R., Williams, D.L., and Kochanek, P.M. (2005). Enhanced oxidative stress in iNOS-deficient mice after traumatic brain injury: support for a neuroprotective role of iNOS. J. Cereb. Blood Flow Metab. 25, 673-684.

Carlos, T.M., Clark, R.S.B., Franicola-Higgins, D., Schiding, J.K., and Kochanek, P.M. (1997). Expression of endothelial adhesion molecules and recruitment of neutrophils after traumatic brain injury. J. Leukoc. Biol. 61, 279-285.

Clark, R.S., Kochanek, P.M., Schwarz, M.A., Schiding, J.K., Turner, D.S., Chen, M., Carlos, T.M., and Watkins, S.C. (1996). Inducible nitric oxide synthase expression in cerebrovascular smooth muscle and neutrophils after traumatic brain injury in immature rats. Pediatr. Res. 39, 784-790.

Cullis, E.R., Kalber, T.L., Ashton, S.E., Cartwright, J.E., Griffiths, J.R., Ryan, A.J., and Robinson, S.P. (2006). Tumour overexpression of inducible nitric oxide synthase (iNOS) increases angiogenesis and may modulate the anti-tumour effects of the vascular disrupting agent ZD6126. Microvasc. Res. 71, 76-84.

Detre, J.A., Leigh, J.S., Williams, D.S., and Koretsky, A.P. (1992). Perfusion Imaging. Magn. Reson. Med. 23, 37-45.

Foley, L.M., Hitchens, T.K., Kochanek, P.M, Melick, J.A., Jackson, E.K., and Ho, C. (2005). Murine orthostatic response during prolonged vertical studies: effect on cerebral blood flow measured by arterial spin-labeled MRI. Magn. Reson. Med. 54, 798-806.

Gahm, C., Holmin, S., and Mathiesen, T. (2000). Temporal profiles and cellular sources of three nitric oxide synthase isoforms in the brain after experimental contusion. Neurosurgery 46, 169-177.

Hayashi, T., Noshita, N., Sugawara, T., and Chan, P.H. (2003). Temporal profile of angiogenesis and expression of related genes in the brain after ischemia. J. Cereb. Blood Flow Metab. 23, 166-180.

Hendrich, K.S., Kochanek, P.M., Williams, D.S., Schiding, J.K., Marion, D.W., and Ho, C. (1999). Early perfusion after controlled cortical impact in rats: quantification by arterial spin-labeled MRI and the influence of spin-lattice relaxation time heterogeneity. Magn. Reson. Med. 42, 673-681.

Herscovitch, P., and Raichle, M.E. (1985). What is the correct value for the brain-blood partition coefficient for water? . Cereb. Blood Flow Metab. 5, 65-69.

Hovda, D.A., Lee, S.M., Smith, M.L., Von Stuck, S., Bergsneider, M., Kelly, D., Shalmon, E., Martin, N., Caron, M., and Mazziotta, J. (1995). The neurochemical and metabolic cascade following brain injury: moving from animal models to man. J. Neurotrauma 12, 903-906.

Huang, Z., Huang, P.L., Panahian, N., Dalkara, T., Fishman, M.C. and Moskowitz, M.A. (1994). Effects of cerebral ischemia in mice deficient in neuronal nitric oxide synthase. Science 265, 1883-1885. 


\section{INDUCIBLE NITRIC OXIDE SYNTHASE AND CEREBRAL BLOOD FLOW AFTER TBI}

Iadecola, C., Zhang, F., and Xu, X. (1995). Inhibition of inducible nitric oxide synthase ameliorates cerebral ischemic damage. Am. J. Physiol. Regul. Integr. Comp. Physiol. 268, 286-292.

Iadecola, C., Zhang, F., Casey, R., Clark, H.B., and Ross, M. E. (1996). Inducible nitric oxide synthase gene expression in vascular cells after transient focal ischemia. Stroke 27, $1373-1380$.

Iadecola, C., Zhang, F., Casey, R., Nagayama, M., and Ross, M.E. (1997). Delayed reduction of ischemic brain injury and neurological deficits in mice lacking the inducible nitric oxide synthase gene. J. Neurosci. 17, 9157-9164.

Jafarian-Tehrani, M., Louin, G., Royo, N.C., Besson, V.C., Bohme, G.A., Plotkine, M., and Marchand-Verrecchia, C. (2005). 1400w, a potent selective inducible NOS inhibitor, improves histopathological outcome following traumatic brain injury in rats. Nitric Oxide 12, 61-69.

Kapinya, K.J., Lowl, D., Futterer, C., Maurer, M., Waschke, K.F., Isaev, N.K., and Dirnagl, U. (2002). Tolerance against ischemic neuronal injury can be induced by volatile anesthetics and is inducible NO synthase dependent. Stroke 33, 1889-1898.

Kawano, T., Kunz, A., Abe, T., Girouard, H., Anrather, J, Zhou, P., and Iadecola, C. (2007). iNOS-derived NO and nox2-derived superoxide confer tolerance to excitotoxic brain injury through peroxynitrite. J. Cereb. Blood Flow Metab. 27, $1453-1462$.

Louin, G., Marchand-Verrecchia, C., Palmier, B., Plotkine, M., and Jafarian-Tehrani, M. (2006). Selective inhibition of inducible nitric oxide synthase reduces neurological deficit but not cerebral edema following traumatic brain injury. Neuropharmacology 50, 182-190.

Okamoto, H., Ito, O., Roman, R.J., and Hudetz, A.G. (1998). Role of inducible nitric oxide synthase and cyclooxygenase2 in endotoxin-induced cerebral hyperemia. Stroke 29, 1209-1218.

Pearse, D.D., Chatzipanteli, K., Marcillo, A.E., Bunge, M.B., and Dietrich, W.D. (2003). Comparison of iNOS inhibition by antisense and pharmacological inhibitors after spinal cord injury. J. Neuropathol. Exp. Neurol. 62, 1096-1107.

Petrov, T., Page, A.B., Owen, C.R., and Rafols, J.A. (2000). Expression of the inducible nitric oxide synthase in distinct cellular types after traumatic brain injury: an in situ hybridization and immunocytochemical study. Acta Neuropathol. 100, 196-204.

Sidman, R.L., Angevine, J.B., and Taber Pierce, E. (1971). Atlas of the Mouse Brain and Spinal Cord. Harvard University Press: Cambridge, MA.

Sinz, E.H., Kochanek, P.M., Dixon, C.E., Clark, R.S., Carcillo, J.A., Schiding, J.K., Chen, M., Wisniewski, S.R., Carlos, T.M., Williams, D., DeKosky, S.T., Watkins, S.C., Marion,
D.W., and Billiar, T.R. (1999). Inducible nitric oxide synthase is an endogenous neuroprotectant after traumatic brain injury in rats and mice. J. Clin. Invest. 104, 647-656.

Smith, D.H., Soares, H.D., Pierce, J.S., Perlman, K.G., Saatman, K.E., Meaney, D.F., Dixon, C.E., and McIntosh, T.K. (1995). A model of parasagittal controlled cortical impact in the mouse: cognitive and histopathologic effects. J. Neurotrauma 12, 169-178.

Steiner, J., Rafols, D., Park, H.K., Katar, M.S., Rafols, J.A. and Petrov, TH. (2004). Attenuation of iNOS mRNA exacerbates hypoperfusion and upregulates endothelin-1 expression in hippocampus and cortex after brain trauma. Nitric Oxide 10, $162-169$

Stoffel, M., Rinecker, M., Plesnila, N., Eriskat, J., and Baethmann, A. (2000). Attenuation of secondary lesion growth in the brain after trauma by selective inhibition of the inducible NO-synthase. Acta Neurochir. Suppl. 76, 357-358.

Wada, K., Chatzipanteli, K., Busto, R., and Dietrich, W.D. (1998a). Role of nitric oxide in traumatic brain injury in the rat. J. Neurosurg. 89, 807-818.

Wada, K., Chatzipanteli, K., Kraydieh, S., Busto, R., and Dietrich, W.D. (1998b). Inducible nitric oxide synthase expression after traumatic brain injury and neuroprotection with aminoguanidine treatment in rats. Neurosurgery 43, 14271436.

Whalen, M.J., Carlos, T.M., Dixon, C.E., Schiding, J.K., Clark, R.S., Baum, E., Yan, H. Q., Marion, D.W., and Kochanek, P.M. (1999). Effect of traumatic brain injury in mice deficient in intercellular adhesion molecule-1: assessment of histopathologic and functional outcome. J. Neurotrauma 16, 299-309.

Williams, D.S., Detre, J.A., Leigh, J.S., and Koretsky, A.P. (1992). Magnetic resonance imaging of perfusion using spin inversion of arterial water. Proc. Natl. Acad. Sci. USA 89, 212-216.

Willmot, M., Gibson, C., Gray, L., Murphy, S., and Bath, P. (2005). Nitric oxide synthase inhibitors in experimental ischemic stroke and their effects on infarct size and cerebral blood flow: a systematic review. Free Radic. Biol. Med. 39, 412-425.

Zhao, P., and Zuo, Z. (2004). Isoflurane preconditioning induces neuroprotection that is inducible nitric oxide synthasedependent in neonatal rats. Anesthesiology 101, 695-702.

Zhang, W., Williams D.S., and Koretsky, A.P. (1993). Measurement of rat brain perfusion by NMR using spin labeling of arterial water: in vivo determination of the degree of spin labeling. Magn. Reson. Med. 29, 416-421.

Zhang, W., Silva, A.C., Williams, D.S., and Koretsky, A.P. (1995). NMR measurement of perfusion using arterial spin labeling without saturation of macromolecular spins. Magn. Reson. Med. 33, 370-376. 


\section{FOLEY ET AL.}

Zhang, R., Wang, Y., Zhang, L., Zhang, Z., Tsang, W., Lu, M., Zhang, L., and Chopp, M. (2002). Sildenafil (Viagra) induces neurogenesis and promotes functional recovery after stroke in rats. Stroke 33, 2675-2680.

Zhu, D.Y., Liu, S.H., Sun, H.S., and Lu, Y.M. (2003). Expression of inducible nitric oxide synthase after focal cerebral ischemis stimulates neurogenesis in the adult rodent dentate gyrus. J. Neurosci. 23, 223-229.
Address reprint requests to:

Patrick M. Kochanek, M.D. Safar Center for Resuscitation Research Department of Critical Care Medicine University of Pittsburgh School of Medicine 3434 Fifth Avenue Pittsburgh, PA 15260

E-mail: kochanekpm@ccm.upmc.edu 


\section{This article has been cited by:}

1. Shein Steven L., Shellington David K., Exo Jennifer L., Jackson Travis C., Wisniewski Stephen R., Jackson Edwin K., Vagni Vincent A., Bayır Hülya, Clark Robert S.B., Dixon C. Edward, Janesko-Feldman Keri L., Kochanek Patrick M.. 2014. Hemorrhagic Shock Shifts the Serum Cytokine Profile from Pro- to Anti-Inflammatory after Experimental Traumatic Brain Injury in Mice. Journal of Neurotrauma 31:16, 1386-1395. [Abstract] [Full Text HTML] [Full Text PDF] [Full Text PDF with Links]

2. Eric E Abrahamson, Lesley M Foley, Steven T DeKosky, T Kevin Hitchens, Chien Ho, Patrick M Kochanek, Milos D Ikonomovic. 2013. Cerebral blood flow changes after brain injury in human amyloid-beta knock-in mice. Journal of Cerebral Blood Flow \& Metabolism 33, 826-833. [CrossRef]

3. Bhavani P. Thampatty, Megan M Klamerus, Patrick J. Oberly, Kerri L. Feldman, Michael J. Bell, Elizabeth C. Tyler-Kabara, P. David Adelson, Robert S.B. Clark, Patrick M. Kochanek, Samuel M. Poloyac. 2013. Hypothermia Decreases Cerebrospinal Fluid Asymmetric Dimethylarginine Levels in Traumatic Brain Injury Children. Pediatric Critical Care Medicine 1. [CrossRef]

4. Lesley M Foley, Alia M Iqbal O'Meara, Stephen R Wisniewski, T Kevin Hitchens, John A Melick, Chien Ho, Larry W Jenkins, Patrick M Kochanek. 2013. MRI assessment of cerebral blood flow after experimental traumatic brain injury combined with hemorrhagic shock in mice. Journal of Cerebral Blood Flow \& Metabolism 33, 129-136. [CrossRef]

5. Jun Zhou, Peng-Fei Wu, Fang Wang, Jian-Guo Chen. 2012. Targeting gaseous molecules to protect against cerebral ischaemic injury: Mechanisms and prospects. Clinical and Experimental Pharmacology and Physiology 39:10.1111/cep.2012.39.issue-6, 566-576. [CrossRef]

6. En Mu, Renyu Ding, Xin An, Xin Li, Song Chen, Xiaochun Ma. 2012. Heparin attenuates lipopolysaccharide-induced acute lung injury by inhibiting nitric oxide synthase and TGF- $\beta$ /Smad signaling pathway. Thrombosis Research 129, 479-485. [CrossRef]

7. Yijen Wu, Qing Ye, Haosen Zhang, T Hitchens, Chien HoOther Non-Stem Cell Therapies for Cellular Tracking-Inflammatory Cell Tracking 335-352. [CrossRef]

8. W. Wouter Oosterlinck, T. Dresselaers, V. Geldhof, A. Van Santvoort, W. Robberecht, P. Herijgers, U. Himmelreich. 2011. Response of mouse brain perfusion to hypo- and hyperventilation measured by arterial spin labeling. Magnetic Resonance in Medicine 66, 802-811. [CrossRef]

9. Bingwen Zheng, Philip Teck Hock Lee, Xavier Golay. 2010. High-sensitivity cerebral perfusion mapping in mice by kbGRASEFAIR at 9.4 T. NMR in Biomedicine 23, 1061-1070. [CrossRef]

10. Ramona R. Hicks, Stephanie J. Fertig, Rebecca E. Desrocher, Walter J. Koroshetz, Joseph J. Pancrazio. 2010. Neurological Effects of Blast Injury. The Journal of Trauma: Injury, Infection, and Critical Care 68, 1257-1263. [CrossRef]

11. Catherine P. Claus, Kyoko Tsuru-Aoyagi, Hita Adwanikar, Breset Walker, William Whetstone, Linda J. Noble-Haeusslein. 2010. Age Is a Determinant of Leukocyte Infiltration and Loss of Cortical Volume after Traumatic Brain Injury. Developmental Neuroscience . [CrossRef]

12. Cornelia Lundblad, Per-Olof Grände, Peter Bentzer. 2009. Hemodynamic and Histological Effects of Traumatic Brain Injury in eNOS-Deficient Mice. Journal of Neurotrauma 26:11, 1953-1962. [Abstract] [Full Text HTML] [Full Text PDF] [Full Text PDF with Links]

13. Valerie C Besson. 2009. Drug targets for traumatic brain injury from poly(ADP-ribose)polymerase pathway modulation. British Journal of Pharmacology 157:10.1111/bph.2009.157.issue-5, 695-704. [CrossRef]

14. Alia Marie Dennis, M. Lee Haselkorn, Vincent A. Vagni, Robert H. Garman, Keri Janesko-Feldman, Hülya Bayır, Robert S.B. Clark, Larry W. Jenkins, C. Edward Dixon, Patrick M. Kochanek. 2009. Hemorrhagic Shock after Experimental Traumatic Brain Injury in Mice: Effect on Neuronal Death. Journal of Neurotrauma 26:6, 889-899. [Abstract] [Full Text PDF] [Full Text PDF with Links]

15. Stanislav I. Svetlov, Stephen F. Larner, Daniel R. Kirk, Joseph Atkinson, Ronald L. Hayes, Kevin K.W. Wang. 2009. Biomarkers of Blast-Induced Neurotrauma: Profiling Molecular and Cellular Mechanisms of Blast Brain Injury. Journal of Neurotrauma 26:6, 913-921. [Abstract] [Full Text PDF] [Full Text PDF with Links] 